Bull. Korean Math. Soc. 46 (2009), No. 3, pp. 545-551

DOI 10.4134/BKMS.2009.46.3.545

\title{
MAPS IN MINIMAL INJECTIVE RESOLUTIONS OF MODULES
}

\author{
KISUK LEE
}

\begin{abstract}
We investigate the behavior of maps in minimal injective resolution of an $A$-module $M$ when $\mu_{t}(\mathfrak{m}, M)=1$ for some $t$, and we develop slightly the fact that a module of type 1 is Cohen-Macaulay.
\end{abstract}

\section{Introduction}

All rings $(A, \mathfrak{m})$ we consider in this paper are assumed to be Noetherian local, and all modules are assumed to be finite and unital.

Let $M$ be a finitely generated module of dimension $d$ over a Noetherian local ring $(A, \mathfrak{m})$. The $i$-th Bass number of $M$ at a prime ideal $\mathfrak{p}$ of $A$, denoted $\mu_{i}(\mathfrak{p}, M)$, is defined to be $\operatorname{dim}_{k(\mathfrak{p})} \operatorname{Ext}_{A_{\mathfrak{p}}}^{i}\left(k(\mathfrak{p}), M_{\mathfrak{p}}\right)$, where $k(\mathfrak{p})=A_{\mathfrak{p}} / \mathfrak{p} A_{\mathfrak{p}}$ : we set $\mu_{i}(M)=\mu_{i}(\mathfrak{m}, M)$ for brevity. The type of $M$, denoted by $r(M)$, is defined to be $\mu_{d}(M)$. One of the interesting studies on the types of rings is to investigate the conditions which make $A$ Cohen-Macaulay when a type of $A$ is known ([1, $3,5,7,8,9$, and 12]).

It is well known ([2]) that Cohen-Macaulay rings $A$ with $r(A)=1$ are Gorenstein. Vasconcelos ([13]) conjectured that the condition $r(A)=1$ is sufficient for $A$ to be Gorenstein, i.e., the condition " $A$ is Cohen-Macaulay" can be omitted. In [4], Foxby proved this conjecture for essentially equicharacteristic rings using a version of the Intersection Theorem. The conjecture was proven in general by Roberts ([12]): he showed that local rings of type one are CohenMacaulay, (and hence Gorenstein) using a minimal free resolution of a dualizing complex.

The purpose of this article is to develop slightly the above fact using the behavior of maps in minimal injective resolutions.

In the next section, we first investigate the behavior of maps in minimal injective resolution $\left(I^{\bullet}, \phi^{\bullet}\right)$ of a module $M$ when the $t$-th Bass number $\mu_{t}(\mathfrak{m}, M)=$

Received July 9, 2008.

2000 Mathematics Subject Classification. 13H10, 13C14, 13D02.

Key words and phrases. Bass numbers, minimal injective resolutions, type of modules, Cohen-Macaulay rings.

The author was supported by 2007 Sookmyung Women's University Research Grant. 
1. More precisely, we prove that when $\mu_{t}(\mathfrak{m}, M)=1$ for some positive integer $t$ and $\mathfrak{p}$ is any prime ideal with $\operatorname{dim} M_{\mathfrak{p}}=d-1$,

(1) either every entry of $f_{t-1}$, or every entry of $f_{t}$ is contained in $\mathfrak{p}$ where $f_{i}$ is a restriction map of $\phi^{i}$ to $E(A / \mathfrak{m})^{\mu_{i}(\mathfrak{m})}$ for each $i$, and

(2) if $\mu_{t-1}(\mathfrak{p}, M) \neq 0$, then every entry of $f_{t-1}$ is contained in $\mathfrak{p}$. From this fact and New Intersection Theorem, we can prove that if $\mu_{t}(\mathfrak{q}, M)=1$ for some $\mathfrak{q} \in \operatorname{Supp}(M)$ and some $t \leq \operatorname{dim} M_{\mathfrak{q}}$, then $\mu_{j}(\mathfrak{q}, M)=0$ for all $j<t$. As a result of this theorem, the well-known fact ([12]) that 'a ring of type 1 is Gorenstein' can be obtained obviously.

\section{Main theorem}

In this section, we investigate the behavior of maps in minimal injective resolution of an $A$-module $M$ when $\mu_{t}(\mathfrak{m}, M)=1$ for some $t$, and then using this and the New Intersection Theorem, we prove Theorem 2.5, from which we obtain the old theorem: 'The module of type 1 is Cohen-Macaulay'.

We first recall the definition of Bass numbers of modules: Let $(A, \mathfrak{m}, k)$ be a Noetherian local ring and $M$ a finite $A$-module of dimension $d$. For a prime ideal $\mathfrak{p}$, the $i$-th Bass number of $M$ at $\mathfrak{p}$ is defined by $\operatorname{dim}_{k(\mathfrak{p})} \operatorname{Ext}_{A_{\mathfrak{p}}}^{i}\left(k(\mathfrak{p}), M_{\mathfrak{p}}\right)$, and denoted $\mu_{i}(\mathfrak{p}, M)$. In particular, the $d$-th Bass number $\mu_{d}(\mathfrak{m}, M)$ is called the type of $M$.

It is known that $\mu_{i}(\mathfrak{p}, M)$ is equal to the number of copies of $E(A / \mathfrak{p})$ which appear in $I^{i}$ as a direct summand, where $I^{\bullet}$ is the minimal injective resolution of $M$ and $E(A / \mathfrak{p})$ denote the injective hull of $A / \mathfrak{p}$, i.e., if

$$
\left(I^{\bullet}, \phi^{\bullet}\right): 0 \rightarrow M \rightarrow I^{0} \rightarrow I^{1} \rightarrow \cdots \rightarrow I^{i} \rightarrow \cdots
$$

is a minimal injective resolution of $M$, then each $I^{i}$ is $\oplus_{\mathfrak{p} \in \operatorname{Supp}(M)} E(A / \mathfrak{p})^{\mu_{i}(\mathfrak{p}, M)}$.

The following fact is well-known, and its proof is elementary:

Fact 2.1. For prime ideals $\mathfrak{p}$ and $\mathfrak{q}$ of a Noetherian local ring $A$,

(1) $\operatorname{Hom}(A / \mathfrak{p}, E(A / \mathfrak{q}))=0$ if $\mathfrak{p} \not \subset \mathfrak{q}$, and $\operatorname{Hom}(A / \mathfrak{p}, E(A / \mathfrak{q})) \cong E_{A / \mathfrak{p}}(A / \mathfrak{q})$ otherwise.

(2) $\operatorname{Hom}(E(A / \mathfrak{p}), E(A / \mathfrak{q}))=0$ if $\mathfrak{p} \not \subset \mathfrak{q}$.

In a minimal injective resolution $\left(I^{\bullet}, \phi^{\bullet}\right)$ of $M$, let $f_{i}$ be a restriction map of $\phi^{i}$ to $E(A / \mathfrak{m})^{\mu_{i}(\mathfrak{m})}$ for each $i$. For a fixed $\mathfrak{p} \in \operatorname{Supp}(M)$ such that $\operatorname{dim} M_{\mathfrak{p}}=$ $d-1$, by applying $\operatorname{Hom}(A / \mathfrak{p}, \bullet)$ to $I^{\bullet}$ and using Fact 2.1 , we have a complex of injective $A / \mathfrak{p}$-modules:

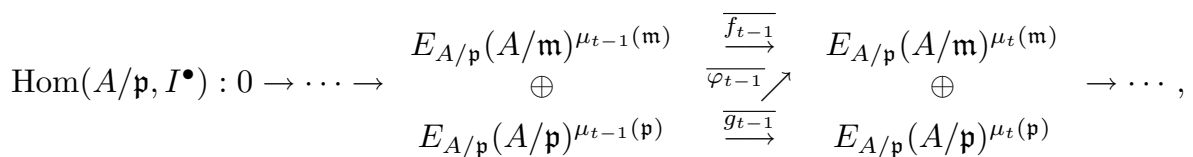

where $\overline{f_{t-1}}: E_{A / \mathfrak{p}}(A / \mathfrak{m})^{\mu_{t-1}(\mathfrak{m})} \rightarrow E_{A / \mathfrak{p}}(A / \mathfrak{m})^{\mu_{t}(\mathfrak{m})}, \overline{\varphi_{t-1}}: E_{A / \mathfrak{p}}(A / \mathfrak{p})^{\mu_{t-1}(\mathfrak{p})} \rightarrow$ $E_{A / \mathfrak{p}}(A / \mathfrak{m})^{\mu_{t}(\mathfrak{m})}$, and $\overline{g_{t-1}}: E_{A / \mathfrak{p}}(A / \mathfrak{p})^{\mu_{t-1}(\mathfrak{p})} \rightarrow E_{A / \mathfrak{p}}(A / \mathfrak{p})^{\mu_{t}(\mathfrak{p})}$. 
With the notations described above, we have Proposition 2.3 below, which shows that there is some restriction on the maps of $f_{i}$. The proof of the proposition uses the following fact:

Fact 2.2 ([4] or $[6])$. Let $M$ be a finitely generated $A$-module. Suppose $\operatorname{ht}(\mathfrak{q} / \mathfrak{p})=1$ for prime ideals $\mathfrak{p}$ and $\mathfrak{q}$. Then we have

$$
\mu_{i}(\mathfrak{p}, M) \leq \mu_{i+1}(\mathfrak{q}, M)
$$

Proposition 2.3. Let $(A, \mathfrak{m}, k)$ be a Noetherian local ring and $M$ a finitely generated A-module of dimension d. Suppose that $\mu_{t}(\mathfrak{m}, M)=1$ for some positive integer $t$, and let $\mathfrak{p}$ be any prime ideal with $\operatorname{dim} M_{\mathfrak{p}}=d-1$. Then

(1) either every entry of $f_{t-1}$, or every entry of $f_{t}$ is contained in $\mathfrak{p}$, and

(2) if $\mu_{t-1}(\mathfrak{p}, M) \neq 0$, then every entry of $f_{t-1}$ is contained in $\mathfrak{p}$.

In particular, if $\mu_{d}(\mathfrak{m}, M)=1$, then every entry of $f_{d-1}$ is contained in $\mathfrak{p}$ with $\operatorname{dim} M_{\mathfrak{p}}=d-1$.

Proof. Let $\overline{f_{t-1}}=\left(\overline{a_{1}}, \ldots, \overline{a_{r}}\right)^{T}$, where $r=\mu_{t-1}(\mathfrak{m}, M)$, and $(-)^{T}$ denotes the transpose matrix.

For the part (1), suppose that $\overline{a_{i}} \neq 0$, i.e., $a_{i} \notin \mathfrak{p}$ for some $i$. Then $A / \mathfrak{p} \stackrel{\overline{a_{i}}}{\longleftrightarrow} A / \mathfrak{p}$ is injective and so $E_{A / \mathfrak{p}}(A / \mathfrak{m}) \stackrel{\overline{a_{i}}}{\longrightarrow} E_{A / \mathfrak{p}}(A / \mathfrak{m})$ is surjective since $\operatorname{Hom}_{A}(-, E(A / \mathfrak{m}))$ is an exact functor and $\operatorname{Hom}_{A}(A / \mathfrak{p}, E(A / \mathfrak{m}))=E_{A / \mathfrak{p}}(A / \mathfrak{m})$. Thus $\overline{f_{t-1}}: E_{A / \mathfrak{p}}(A / \mathfrak{m})^{\mu_{t-1}(\mathfrak{m})} \rightarrow E_{A / \mathfrak{p}}(A / \mathfrak{m})$ is also surjective, and so $\overline{f_{t}}$ must be a zero map since $\operatorname{Hom}\left(A / \mathfrak{p}, I^{\bullet}\right)$ is a complex, i.e., every entry of $f_{t}$ is contained in $\mathfrak{p}$.

For the part $(2)$, suppose that $\mu_{t-1}(\mathfrak{p}, M) \neq 0$. Then $\mu_{t-1}(\mathfrak{p}, M)=1$ by Fact 2.2 since $\mu_{t}(\mathfrak{m}, M)=1$. Suppose to the contrary that $\overline{a_{i}} \neq 0$, i.e., $a_{i} \notin \mathfrak{p}$ for some $i$. Then by the same reason as (1), $\overline{f_{t-1}}$ is surjective.

Since $(t-1)$-th cohomology $\operatorname{Ext}_{A}^{t-1}(A / \mathfrak{p}, M)$ is finitely generated and

$$
E_{A / \mathfrak{p}}(A / \mathfrak{p})^{\mu_{t-2}(\mathfrak{p})} \rightarrow E_{A / \mathfrak{p}}(A / \mathfrak{p})
$$

is a zero map ([10, Proposition 2.5 in Chapter 1$])$, there are finite elements in $E_{A / \mathfrak{p}}(A / \mathfrak{p})$ such that the second component of every element of $\operatorname{Ker} \overline{\phi^{t-1}}$ is generated by them. Since $E_{A / \mathfrak{p}}(A / \mathfrak{p}) \cong Q(A / \mathfrak{p})$, which is the field of quotients of $A / \mathfrak{p}$, we may choose some $x$ in $\mathfrak{m} / \mathfrak{p}$ such that

$$
\operatorname{Ker} \overline{\phi^{t-1}} \subseteq E_{A / \mathfrak{p}}(A / \mathfrak{m})^{\mu_{t-1}(\mathfrak{m})} \oplus \frac{1}{x} A / \mathfrak{p} .
$$

Since $\overline{f_{t-1}}$ is surjective, $\overline{\varphi_{t-1}}\left(\frac{1}{x^{2}}\right)=\overline{f_{t-1}}(a)$ for some $a \in E_{A / \mathfrak{p}}(A / \mathfrak{m})^{\mu_{t-1}(\mathfrak{m})}$. Therefore,

$$
\left(-a, \frac{1}{x^{2}}\right) \in \operatorname{Ker}\left(\overline{f_{t-1}} \oplus \overline{\varphi_{t-1}}\right) \subseteq \operatorname{Ker} \overline{\phi^{t-1}} \subseteq E_{A / \mathfrak{p}}(A / \mathfrak{m})^{\mu_{t-1}(\mathfrak{m})} \oplus \frac{1}{x} A / \mathfrak{p},
$$

i.e., $\frac{1}{x^{2}} \in \frac{1}{x} A / \mathfrak{p}$, which is a contradiction. Hence each $a_{i}$ of $f_{t-1}$ belongs to $\mathfrak{p}$.

For the last part of $(2)$, we need to show that $\mu_{d-1}(\mathfrak{p}, M) \neq 0$ for a prime $\mathfrak{p}$ with $\operatorname{dim} M_{\mathfrak{p}}=d-1$. Indeed, $\mu_{d-1}(\mathfrak{p}, M) \neq 0$ by Fact 2.2 since $\mu_{0}\left(\mathfrak{p}_{0}, M\right)$ 
$\neq 0$, where a prime ideal $\mathfrak{p}_{0}$ is minimal over $\operatorname{ann}(M)$, i.e., $\mathfrak{p}_{0} \in \operatorname{Ass}(M)$ and $\operatorname{ht}\left(\mathfrak{p} / \mathfrak{p}_{0}\right)=d-1$.

We note the following easy fact:

Lemma 2.4. Let $A$ be a catenary local domain of dimension $d$ and $M$ be a finitely generated $A$-module. If $M_{\mathfrak{p}}=0$ for each prime ideal $\mathfrak{p}$ with $\mathrm{ht}(\mathfrak{p})=d-1$, then $\ell(M)<\infty$.

Proof. Let $\mathfrak{q}$ be a prime ideal of $h t(\mathfrak{m} / \mathfrak{q})=1$. Since $A$ is a catenary domain, we know $\operatorname{ht}(\mathfrak{q})=d-1$. Then $M_{\mathfrak{q}}=0$ by assumption, which concludes that $\operatorname{Supp}(M)=\{\mathfrak{m}\}$. Hence $M$ has a finite length.

To prove the main theorem in this section, the New Intersection Theorem is used:

New Intersection Theorem ([11]). Let $F_{\bullet}: 0 \rightarrow F_{k} \rightarrow \cdots \rightarrow F_{0} \rightarrow 0$ be a non-exact complex of free A-modules with homologies of finite length. Then

$$
\operatorname{dim} A \leq k .
$$

We also recall the facts that $\mu_{i}(\mathfrak{m}, M)=\mu_{i}(\hat{\mathfrak{m}}, \hat{M})$, where $\hat{M}$ denotes the $\mathfrak{m}$ adic completion of $M$, and $\operatorname{depth} M=\inf \left\{i: \mu_{i}(\mathfrak{m}, M) \neq 0\right\}$ and $\operatorname{inj\operatorname {dim}} M=$ $\sup \left\{i: \mu_{i}(\mathfrak{m}, M) \neq 0\right\}$.

Now we have the main theorem.

Theorem 2.5. Let $(A, \mathfrak{m}, k)$ be a Noetherian local ring of dimension $n$ and $M$ a finitely generated $A$-module of dimension d. If $\mu_{t}(\mathfrak{q}, M)=1$ for some $\mathfrak{q} \in \operatorname{Supp}(M)$ and some $t \leq \operatorname{dim} M_{\mathfrak{q}}$, then $\mu_{j}(\mathfrak{q}, M)=0$ for all $j<t$.

Proof. Localizing at $\mathfrak{q}$, we may assume that $\mathfrak{q}=\mathfrak{m}$. As usual, we may also assume that $A$ is complete since $\mu_{i}(\mathfrak{m}, M)=\mu_{i}(\hat{\mathfrak{m}}, \hat{M})$. We use an induction on the dimension of $M$. If $\operatorname{dim} M=0$, then we are through. Now suppose that $\operatorname{dim} M=d>0$ and $t \leq d$. We will construct a non-exact complex of free modules with homologies of finite length so that we apply the New Intersection Theorem to complete the proof.

As in the proof of Proposition 2.3, let

$$
\left(I^{\bullet}, \phi^{\bullet}\right): 0 \rightarrow M \rightarrow I^{0} \rightarrow I^{1} \rightarrow \cdots \rightarrow I^{i} \rightarrow \cdots,
$$

be a minimal injective resolution of $M$, where $I^{i}=\oplus_{\mathfrak{p} \in \operatorname{Supp}(M)} E(A / \mathfrak{p})^{\mu_{i}(\mathfrak{p}, M)}$. Let $f_{j}$ be a restriction of $\phi^{j}$ to $E(A / \mathfrak{m})^{\mu_{j}(\mathfrak{m})}$.

Suppose that every entry of $f_{t-1}$ is contained in $\mathfrak{p}$ for all prime ideals $\mathfrak{p}$ with $\operatorname{dim} M_{\mathfrak{p}}=d-1$.

By applying $\operatorname{Hom}_{A}(-, E(A / \mathfrak{m}))$ to $H_{\mathfrak{m}}^{0}\left(I^{\bullet}\right)\left(=\lim _{\rightarrow} \operatorname{Hom}_{A}\left(A / \mathfrak{m}^{r}, I^{\bullet}\right)\right)$, we have

$$
F_{\bullet}: \cdots \rightarrow A \stackrel{f_{t-1}^{T}}{\longrightarrow} A^{\mu_{t-1}(\mathfrak{m})} \rightarrow \cdots \rightarrow A^{\mu_{1}(\mathfrak{m})} \rightarrow A^{\mu_{0}(\mathfrak{m})} \rightarrow 0 .
$$

We note that the $i$-th homology of $F_{\bullet}$ is $H_{\mathrm{m}}^{i}(M)^{\vee}$, where $(-)^{\vee}$ denotes the Matlis dual. Since $A$ is complete, $A / \operatorname{ann}(M) \cong S / J$ such that $\left(S, \mathfrak{m}_{S}\right)$ is a 
Gorenstein local ring, $J$ is an ideal of $S$ and $\operatorname{dim} S=\operatorname{dim} A / \operatorname{ann}(M)=\operatorname{dim} M$. Hence by local duality

$$
H_{\mathfrak{m}}^{i}(M)^{\vee} \cong H_{\mathfrak{m}_{S}}^{i}\left({ }_{s} M\right)^{\vee} \cong \operatorname{Ext}_{S}^{d-i}\left({ }_{s} M, S\right)
$$

For a prime ideal $\mathfrak{p}$ of $A$ with $\operatorname{dim} M_{\mathfrak{p}}=d-1$, since $A / \operatorname{ann}(M) \cong S / J$ and $\operatorname{dim} A / \operatorname{ann}(M)=\operatorname{dim} S$, there is a corresponding prime ideal $\mathfrak{p}_{s}$ of $S$ such that $d-1=\operatorname{dim} M_{\mathfrak{p}}=\operatorname{dim} S_{\mathfrak{p}_{s}}$. We note that

$$
\begin{aligned}
H_{i}\left(F_{\bullet} \otimes A_{\mathfrak{p}}\right) & \cong H_{i}\left(F_{\bullet}\right) \otimes A_{\mathfrak{p}} \cong H_{\mathfrak{m}}^{i}(M)^{\vee} \otimes A_{\mathfrak{p}} \\
& \cong \operatorname{Ext}_{S}^{d-i}(M, S) \otimes S_{\mathfrak{p}_{s}} \cong \operatorname{Ext}_{S_{\mathfrak{p}_{s}}^{d-i}\left(M_{\mathfrak{p}_{s}}, S_{\mathfrak{p}_{s}}\right)} \\
& \cong H_{\mathfrak{p}_{s}}^{i-1}\left(M_{\mathfrak{p}_{s}}\right)^{\vee} .
\end{aligned}
$$

We note that $\operatorname{depth} M_{\mathfrak{p}} \geq t-1$; if $\mu_{t-1}(\mathfrak{p}, M) \neq 0$, then $\operatorname{depth} M_{\mathfrak{p}}=t-1$ by induction hypothesis, and if $\mu_{t-1}(\mathfrak{p}, M)=0$, then $\operatorname{depth} M_{\mathfrak{p}} \geq t$. Thus $\operatorname{depth} M_{\mathfrak{p}_{s}} \geq t-1$, which implies $H_{i}\left(F_{\bullet} \otimes A_{\mathfrak{p}}\right)=0$ for $i-1<t-1$, and so we have the following truncated exact sequence:

$$
F_{i<t} \otimes A_{\mathfrak{p}}: 0 \rightarrow \operatorname{Im}\left(f_{t-1}\right)_{\mathfrak{p}} \rightarrow A_{\mathfrak{p}}^{\mu_{t-1}(\mathfrak{m})} \rightarrow \cdots \rightarrow A_{\mathfrak{p}}^{\mu_{1}(\mathfrak{m})} \rightarrow A_{\mathfrak{p}}^{\mu_{0}(\mathfrak{m})} \rightarrow 0 .
$$

Since an exact sequence $F_{i<t} \otimes A_{\mathfrak{p}}$ splits, $\operatorname{Im}\left(f_{t-1}\right)_{\mathfrak{p}}$ is free. By the assumption, i.e., each element of $f_{t-1}$ belongs to $\mathfrak{p}, \operatorname{Im}\left(f_{t-1}\right)_{\mathfrak{p}}$ must be 0 .

Now let us choose a minimal prime $\mathfrak{q} \in \operatorname{Supp}(M)$ such that $\operatorname{dim} A / \mathfrak{q}=$ $\operatorname{dim} M=d$. We note that $A / \mathfrak{q}$, denoted by $\bar{A}$, is a complete local domain. Let us consider the following non-exact complex of free modules:

$$
\overline{F_{\bullet}}\left(:=A / \mathfrak{q} \otimes F_{i<t}\right): 0 \rightarrow \bar{A}^{\mu_{t-1}(\mathfrak{m})} \rightarrow \cdots \rightarrow \bar{A}^{\mu_{1}(\mathfrak{m})} \rightarrow \bar{A}^{\mu_{0}(\mathfrak{m})} \rightarrow 0 .
$$

For a prime ideal $\mathfrak{p}$ of height $d-1$ in $\bar{A}$, i.e., $\operatorname{dim} M_{\mathfrak{p}}=d-1$,

$$
\begin{aligned}
H_{i}\left(\bar{F}_{\bullet}\right)_{\mathfrak{p}} & \cong H_{i}(\bar{F} \bullet) \otimes \bar{A}_{\mathfrak{p}} \\
& \cong H_{i}\left(\bar{F} \bullet \bar{A}_{\mathfrak{p}}\right) \\
& \cong H_{i}\left(F_{\bullet} \otimes_{A} A / \mathfrak{q} \otimes_{A}(A / \mathfrak{q})_{\mathfrak{p}}\right) \\
& \cong H_{i}\left(\left(F_{\bullet} \otimes_{A} A_{\mathfrak{p}}\right) \otimes_{A_{\mathfrak{p}}} A_{\mathfrak{p}} / \mathfrak{q} A_{\mathfrak{p}}\right),
\end{aligned}
$$

where the last term is 0 since an exact sequence $F_{i<t} \otimes_{A} A_{\mathfrak{p}}$ splits. Hence the length of $H_{i}\left(\bar{F}_{\bullet}\right)$ is finite by Lemma 2.4 .

Since $\bar{F} \bullet$ is not exact (each entry of maps in $\bar{F}$. belongs to $\mathfrak{m}$ ), if $\mu_{i}(\mathfrak{m}, M) \neq$ 0 for some $i$, then by New Intersection Theorem, we have $d=\operatorname{dim} \bar{A} \leq t-1$. This is a contradiction. Hence we have $\mu_{t-1}(\mathfrak{m}, M)=\cdots=\mu_{0}(\mathfrak{m}, M)=0$.

Now suppose that there is a prime ideal $\mathfrak{p}$ with $\operatorname{dim} M_{\mathfrak{p}}=d-1$ such that some entry of $f_{t-1}$ does not belong to $\mathfrak{p}$. Then by Proposition 2.3(1), every entry of $f_{t}$ is contained in $\mathfrak{p}$. We note that depth $M_{\mathfrak{p}} \geq t$ since $\mu_{t-1}(\mathfrak{p}, M)=0$ by Proposition 2.3(2). We also note that $t<d=\operatorname{dim} M$ since if $t=d$, then by Proposition $2.3(2)$, each entry of $f_{t-1}$ is contained in $\mathfrak{p}$, which contradicts the assumption. 
By the similar argument in the first part of proof, we have the following truncated exact sequence:

$$
G_{i<t+1} \otimes A_{\mathfrak{p}}: 0 \rightarrow A_{\mathfrak{p}} \stackrel{f_{t-1}}{\longrightarrow} A_{\mathfrak{p}}^{\mu_{t-1}(\mathfrak{m})} \rightarrow \cdots \rightarrow A_{\mathfrak{p}}^{\mu_{1}(\mathfrak{m})} \rightarrow A_{\mathfrak{p}}^{\mu_{0}(\mathfrak{m})} \rightarrow 0
$$

since depth $M_{\mathfrak{p}} \geq t$ and $\operatorname{Im}\left(f_{t}\right)_{\mathfrak{p}}=0$. Note that $\sum_{i=0}^{t-1}(-1)^{i} \mu_{i}(\mathfrak{m})+(-1)^{t}=0$, which does not depend on the choice of $\mathfrak{p}$. This implies that some entry of $f_{t-1}$ does not belong to $\mathfrak{p}$ for all $\mathfrak{p}$ with $\operatorname{dim} M_{\mathfrak{p}}=d-1$ (if not, we would have $\left.\sum_{i=0}^{t-1}(-1)^{i} \mu_{i}(\mathfrak{m})=0\right)$, and hence $G_{i<t+1} \otimes A_{\mathfrak{p}}$ is exact for all $\mathfrak{p}$ with $\operatorname{dim} M_{\mathfrak{p}}=d-1$.

For a minimal prime $\mathfrak{q} \in \operatorname{Supp}(M)$ such that $\operatorname{dim} A / \mathfrak{q}(=\bar{A})=\operatorname{dim} M=d$, since the following non-exact complex of free modules

$$
\overline{G_{\bullet}}\left(:=A / \mathfrak{q} \otimes F_{i<t+1}\right): 0 \rightarrow \bar{A} \rightarrow \bar{A}^{\mu_{t-1}(\mathfrak{m})} \rightarrow \cdots \rightarrow \bar{A}^{\mu_{1}(\mathfrak{m})} \rightarrow \bar{A}^{\mu_{0}(\mathfrak{m})} \rightarrow 0
$$

has finite homologies, we have $t>\operatorname{dim} \bar{A}=d$ by New Intersection Theorem. This is a contradiction. Hence every entry of $f_{t-1}$ is contained in $\mathfrak{p}$, and $\mu_{j}(\mathfrak{m}, M)=0$ for all $j<t$.

Remark 2.6. In fact, the proof of Theorem 2.5 shows that every entry of a map $f_{t-1}$ in Proposition 2.3 is contained in all $\mathfrak{p}$ with $\operatorname{dim} M_{\mathfrak{p}}=d-1$ if $t \leq d$ and $\mu_{t}(\mathfrak{m}, M)=1$.

In [4, Remark 3.9], Foxby proved that for an essentially equicharacteristic ring $A$, a finite $A$ module of type one is Cohen-Macaulay, and Roberts showed ([12]) that a local ring of type one is Cohen-Macaulay using a minimal free resolution of a dualizing complex. We can get the above results as a corollary of Theorem 2.5 as follows:

Corollary 2.7. Let $(A, \mathfrak{m}, k)$ be a Noetherian local ring of dimension $n$ and $M$ a finitely generated $A$-module of dimension $d$. Suppose $M$ is of type one, i.e., $\mu_{d}(\mathfrak{m}, M)\left(=\operatorname{dim}_{k} \operatorname{Ext}_{A}^{d}(A / \mathfrak{m}, M)\right)=1$. Then $M$ is a Cohen-Macaulay module. In particular, a local ring of type one is Gorenstein.

Proof. If $\mu_{d}(\mathfrak{m}, M)=1$, then $\mu_{j}(\mathfrak{m}, M)=0$ for all $j<d$ by Theorem 2.5, and so $\operatorname{depth} M \geq d=\operatorname{dim} M$. Hence $M$ is a Cohen-Macaulay module. It is well-known that a Cohen-Macaulay local ring of type 1 is Gorenstein.

\section{References}

[1] Y. Aoyama, Complete local $\left(S_{n-1}\right)$ rings of type $n \geq 3$ are Cohen-Macaulay, Proc. Japan Acad. Ser. A Math. Sci. 70 (1994), no. 3, 80-83.

[2] H. Bass, On the ubiquity of Gorenstein rings, Math. Z. 82 (1963), 8-28.

[3] D. Costa, C. Huneke, and M. Miller, Complete local domains of type two are CohenMacaulay, Bull. London Math. Soc. 17 (1985), no. 1, 29-31.

[4] H.-B. Foxby, On the $\mu^{i}$ in a minimal injective resolution. II, Math. Scand. 41 (1977), no. $1,19-44$.

[5] T. Kawasaki, Local rings of relatively small type are Cohen-Macaulay, Proc. Amer. Math. Soc. 122 (1994), no. 3, 703-709. 
[6] J. Koh, M. Kim, and K. Lee, Applications of $S C_{r}$-condition to Bass numbers of modules, in preparation.

[7] K. Lee, A note on types of Noetherian local rings, Bull. Korean Math. Soc. 39 (2002), no. $4,645-652$.

[8] _ On types of Noetherian local rings and modules, J. Korean Math. Soc. 44 (2007), no. 4, 987-995.

[9] T. Marley, Unmixed local rings of type two are Cohen-Macaulay, Bull. London Math. Soc. 23 (1991), no. 1, 43-45.

[10] P. Roberts, Homological Invariants of Modules over Commutative Rings, Presses de l'Université de Montreal, Montréal, Que., 1980.

[11] _ Intersection theorems, Commutative algebra (Berkeley, CA, 1987), 417-436, Math. Sci. Res. Inst. Publ., 15, Springer, New York, 1989.

[12] _ Rings of type 1 are Gorenstein, Bull. London Math. Soc. 15 (1983), no. 1, $48-50$.

[13] W. V. Vasconcelos, Divisor Theory in Module Categories, North-Holland Mathematics Studies, No. 14. Notas de Matematica No. 53. [Notes on Mathematics, No. 53] NorthHolland Publishing Co., Amsterdam-Oxford; American Elsevier Publishing Co., Inc., New York, 1974.

Department of Mathematics

SOOKMYung Women's University

SEOUl 140-742, Korea

E-mail address: kilee@sookmyung.ac.kr 